\title{
Glück und Gesundheit
}

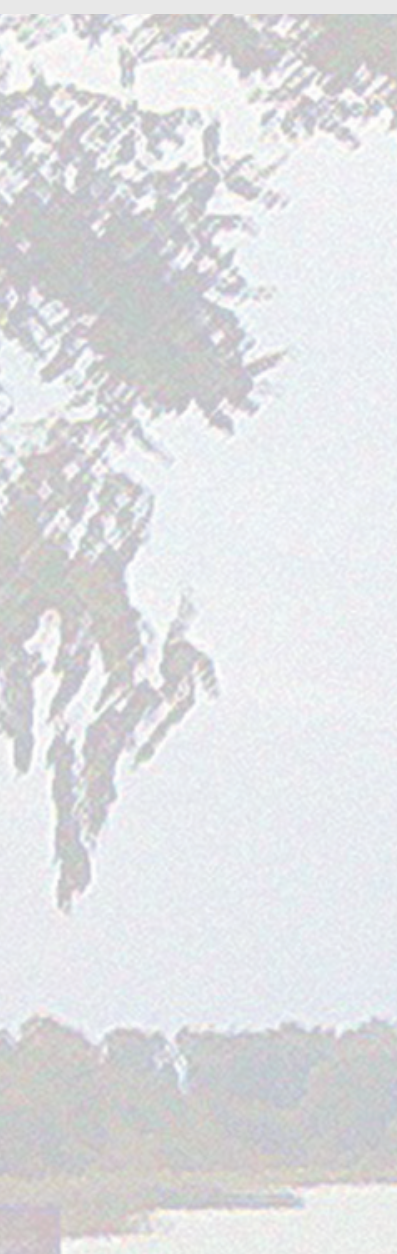

* Heute «dürfen» wir nicht mehr, wir «müssen»-dies erinnert mich an einen Artikel, den ich vor langer Zeit mit dem Titel «Du pouvoir choisir au devoir choisir» geschrieben habe [2]. Gemeint war die Medizin angesichts ihrer begrenzten Finanzierungsmöglichkeiten, ein Thema, das auch anlässlich des Symposiums diskutiert wurde.

1 Binswanger $\mathrm{M}$. Die Tretmühlen des Glücks Freiburg: Herder; 3. Auflage. 2008.

2 Martin J. Du pouvoir choisir au devoir choisir, problématique cruciale et paradoxale des systèmes de santé aujourd'hui.

In: Martin J. Enjeux éthiques en santé publique. Genève: Ed. Médecine et Hygiène; 1991. 119-130.
Anfang September fand am Ufer des Hallwilersees das 5. «Platin-Symposium» der Schweizerischen Gesellschaft für Innere Medizin unter der Leitung ihres ehemaligen Präsidenten Werner Bauer statt. Es trug den Titel «Das Gesundheitswesen - Montage oder Demontage eines Systems?» Nachdem ich über drei Jahrzehnte an vielen Dutzend Runden Tischen, Seminaren, Studientagen und anderen Arbeitsgruppen mitgewirkt habe, deren Ziel die (stets dringende ...) Reform des Gesundheitssystems war, habe ich heute als Pensionär nicht mehr unbedingt das Bestreben, an Versammlungen teilzunehmen, in denen Schöngeister (Ärzte, Politiker, Versicherer und andere) Kostproben ihrer wissenschaftlichen Erkenntnisse, Sorgen, Forderungen, Wahrsagungen geben ... Denn nur zu oft habe ich dabei «Déjà-vu-Erlebnisse». In der noch jungen Tradition des «Platin-Symposiums» ist man aber bemüht, auch Redner aus vollkommen anderen Disziplinen einzuladen. So war in diesem Jahr der Vortrag des Pfarrers Andrea Bianca unter dem Titel «Welche Gesundheit braucht der Mensch?» zu hören. Er sprach über das Verhältnis zwischen Religion, Spiritualität und Gesundheit. Nachdem vor Jahrhunderten die Aufgaben des Geistlichen und des Therapeuten getrennt worden waren, werden der Medizin heute wieder Attribute der Religion zugewiesen (in seinem Schlusswort zu dem Treffen bekräftigte der Philosoph und Leitartikler Ludwig Hasler, sie sei zweifellos die Religion der heutigen Zeit).

Der Wirtschaftsprofessor Mathias Binswanger befasste sich in seinem Referat mit dem Thema Glück, Gesundheit und Wirtschaft. Seiner Ansicht nach gibt es in der heutigen Gesellschaft eine Form von «Laufbändern», die uns dazu zwingen, immer weiter und mehr zu arbeiten. Es ist die bekannte «Tretmühle» (engl. treadmills; das Wort hat seinen Ursprung in den Zuckerrohrplantagen. Die Tretmühle bestand aus einem horizontalen Zylinder, der von einem halben Dutzend Sklaven angetrieben wurde, und erinnert an die Laufräder in Mäusekäfigen, wobei sich die Sklaven ausserhalb des Zylinders befanden). Diese Tretmühlen lassen uns immer länger und schneller treten - es gibt kein Entrinnen! Unser Ziel? Geld, Status und Prestige, sich Genuss leisten können, alle Optionen offen haben. Dabei können wir tagtäglich beobachten, dass dieses Hetzen und Rennen kein Glück bringt, sondern die Gesundheit belastet. Denn man braucht immer mehr, ob von dem einen oder dem anderen. Indem die Globalisierung also nahezu unbegrenzte Chancen zu bieten scheint, fördert sie mit Sicherheit nicht die Gesundheit - und dies ist nur einer ihrer vielen Effekte [1].
Das Problem liegt darin, dass die wachsenden Möglichkeiten, die uns offenstehen, keine wahren Chancen mehr sind, sondern vielmehr Verpflichtungen*. Binswanger gibt dazu den Rat, nicht immer nach dem Besten zu suchen, um wirklich glücklich zu sein. Dies scheint zu überraschen, da ich hier zu Ärzten spreche, zu deren Grundsätzen es gehört, sich für ihre Patienten immer um das Beste zu bemühen. Im persönlichen Leben aber bedeutet die unaufhörliche Suche nach der noch besseren Gelegenheit, die Chance zu verspielen, glücklich zu sein. Er zitiert das Sprichwort «Better be a big frog in a small pond than a small frog in a big pond»-also ist es schöner, ein Bedeutender an einem kleinen Platz zu sein als umgekehrt.

Daher sollten wir unser stetiges «Gut ist nicht gut genug» endlich vergessen. Denn nur eines ist hier wirklich wichtig: Zufriedenheit, Zuversicht, Freude (Glück!), die man aus einem Leben ausserhalb der uns erschöpfenden, ja unser Leben bestimmenden Tretmühlen ziehen kann. Sie sind es, die unsere Gesundheit positiv beeinflussen - was auch in immer mehr wissenschaftlichen Studien belegt wird. Diese Zufriedenheit zu suchen, ist sinnvoller, als Antidepressiva einzunehmen («Wash your blues away with Prozac» ...). Voltaire hatte recht, wenn er seinem Satz, «Die grosse und einzige Lebensfrage, um die man sich kümmern sollte, ist: glücklich zu leben», hinzufügte, «Da es sehr förderlich für die Gesundheit ist, habe ich beschlossen, glücklich zu sein». Pfarrer Bianca sprach von der Notwendigkeit der «Metanoia», des Umdenkens, der Sinnesänderung - des Paradigmenwechsels, um ein Modewort zu verwenden. Eine Frage: Wird der Beweis, dass der Endpunkt des rastlosen Treibens und der Obsessionen in unserer heutigen Zeit nicht das Glück sein kann, eine angemessene Penetranz aufweisen, wider die aktuellen Zeichen der Gesellschaft und der Medien, die alle in die andere Richtung weisen? Und wenn ja, innerhalb von welchem Zeitraum ...?

Noch eine letzte Bemerkung: In seinem Schlusswort betonte Ludwig Hasler, in welchem Masse die Überzeugungskraft und der Enthusiasmus (in gewisser Weise das berufliche Glück), die der Arzt in der Ausübung seines Berufs zeigt, zur Besserung des Gesundheitszustands seiner Patienten beitragen. Das erinnert mich an einen unserer grossen Kollegen, der von 1950-1970 in Lausanne praktizierte und lehrte, Prof. Edouard Jéquier-Doge. Er sprach von Patienten, die nur genesen, um ihrem Arzt eine Freude zu machen.

Dr. Jean Martin, Mitglied der Redaktion und der Nationalen Ethikkommission 